\title{
MONITORING SYSTEM OF RAIL SURFACE CRACK PROPAGATION
}

\author{
Zoltán NYIKES ${ }^{1}$, Dániel TOKODY ${ }^{2}$, Tünde KOVÁCS ${ }^{3}$ \\ 1,2 Óbuda University, Doctoral School of Safety and Security Science, Budapest, Hungary \\ ${ }^{1}$ nyikes.zoltan@phd.uni-obuda.hu \\ 2 tokody.daniel@mav.hu \\ ${ }^{3}$ Óbuda University, Bánki Donát Faculty of Mechanical and Safety Engineering, Budapest, \\ Hungary, kovacs.tunde@bgk.uni-obuda.hu
}

\begin{abstract}
Industrially produced rails can contain some inherent failures without evident damage. If a fracture propagates beyond a critical size, it can lead to breakage. The study of fracture mechanics suggests many different theories for detecting the fracture. Continuous monitoring of the rail surface state is necessary in order to assure uninterrupted and safe transportation.
\end{abstract}

Keywords: crack, fracture toughness, monitoring system.

\section{Introduction}

Railways are a very important infrastructure element for a country. They are one of the most effective means of public transport and goods handling. Since the birth of the railway, the connection between the rail and the railroad has been a key issue. Various forms of damage at the wheel-rail contact can be observed. Fatigue, wear of the rail surface and the formation of cracks result from the continuous load on the surface. Today, different grades of rails are produced, and it is possible to improve their strength by surface heat treatment. The cracks existing or emerging on the track surface can increase due to continuous loading and may lead to fractures. Therefore continuous monitoring is needed.
Currently, the monitoring of railroad tracks is performed visually, which can be very time-consuming. Faults discovered visually are registered manually and then the data is transmitted. The data processing is also done manually.

In accordance with Industry 4.0 requirements, it needs to be integrated with the tools of the digital world. These tools should also be used to support material testing to rapidly and accurately process and transmit data.

\section{Cracks, material discontinuities}

Perfectly failure free material cannot be manufactured by mass production. There can also be small defects or cracks in the manufacturing of rails where loads may cause the cracks to start and grow. In addition, when rails are installed, it's not only 
the rail-wheel connection that creates stress but also the open-air environment; the surface can be subjected to gravel splash and other mechanical impacts that, according to their size and shape, may lead to the occurrence of cracks.

Fracture mechanics can provide a design basis by illuminating the relationship between crack size and load. One way to determine the critical cracking size is to use the fracture toughness equation. The value of fracture toughness depends on the material of the rail. The size of the hazardous crack can be calculated from equation (1). In the equation of fracture toughness, an (m) is the crack half size and $\sigma(\mathrm{MPa})$ is the load stress.

$$
\left.K_{I C}=\sigma \cdot \sqrt{\pi \cdot a} \mid \operatorname{MPa} \sqrt{m}\right]
$$

The stress can be calculated from the size of the contact surface and the load force. In the case of wheel-rail contact, the maximum load can be calculated based on the Hertzian contact stress [1]. The modified form of the stress, the contact surface reduced by the size of the crack (2) [2], where F (N) is the load, the contact surface (ellipse) $\left(\mathrm{mm}^{2}\right), 2 \mathrm{a}_{\mathrm{e}}(\mathrm{mm})$ is the major axis of the ellipse contact surface, $2 b_{e}(\mathrm{~mm})$ the small axis of the contact surface ellipse, e $\left(\mathrm{mm}^{2}\right)$ the measured fracture on the rail surface:

$$
\sigma=\frac{3}{2} \frac{F}{\left(\pi a_{e} b_{e}\right)-e}\left[\frac{N}{m m^{2}}\right]
$$

The crack can be approximated to an elliptical area. The size of the connected surface should be reduced by the size of the surface of the cracking to obtain an approximate value: $K_{I C}=41[\operatorname{MPa} \sqrt{m}]$ is a value for experimentally determined perlite rails (based on the literature) [3].
The rail and wheel contact surface is elliptical and has a large axis $2 \mathrm{a}_{\mathrm{e}}=13 \mathrm{~mm}$, a small axis $2 b_{\mathrm{e}}=10 \mathrm{~mm}$ its area $\mathrm{T}\left(\mathrm{mm}^{2}\right)$ :

$$
T=\pi a_{e} b_{e}=102,5\left[\mathrm{~mm}^{2}\right]
$$

If there is no crack on the rail surface, then $\mathrm{e}=0 \mathrm{~mm}$ the Hertzian stress (2) can be calculated in case of a load of $100 \mathrm{kN}$.

$$
\sigma=\frac{3}{2} \frac{F}{\left(\pi a_{e} b_{e}\right)-e}=1469,86\left[\frac{N}{m^{2}}\right]
$$

By rearranging the fracture toughness equation (1), the maximally tolerated crack size 2a $(\mathrm{mm})$ is obtained:

$$
2 a=2 \cdot \frac{\left(\frac{K_{I C}}{\sigma}\right)^{2}}{\pi}=0,4955[\mathrm{~mm}]
$$

In addition to the given load, the crack size should not be greater than 2a. The cracks can also be approximated by elliptical geometry, with a large axis 2a (mm). Depending on the size of the crack, the Hertzian stress increases, and the degree of damage is proportional. Visual inspection, therefore, requires a camera whose resolution makes it possible to detect the error in the computed range.

\section{Collecting and analyzing data}

In the following section, the information security and testing of the surface finish of the rail are presented.

\subsection{Primary data}

In the case of material testing, large amounts of "raw" data are generated. These are referred to as primary data [4]. These data must be collected, stored and transmitted on the appropriate channel for further evaluation [5]. 


\subsection{Secondary data}

In addition to the rail status data, location coordinates must be stored and transmitted together with the primary data [6]. These data are referred to below as secondary data. Data is stored and transmitted using data fusion.

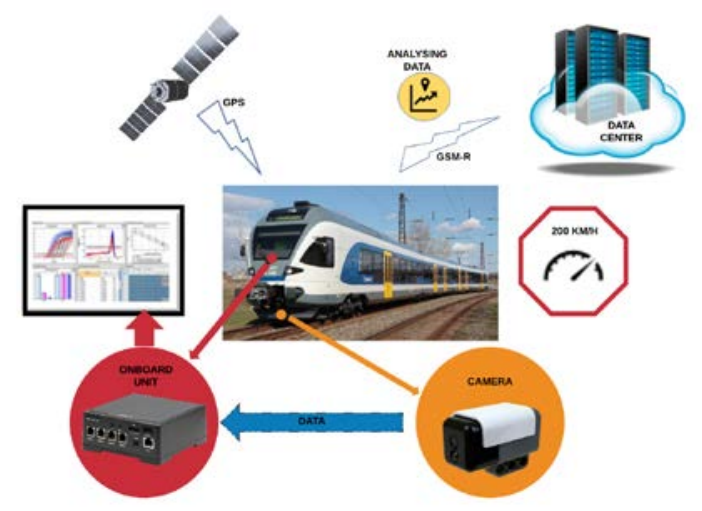

Figure 1. Data analysis and data transfer of the material testing

\subsection{Classification and transmission of data}

The data generated from the railway track and its analysis are different. Due to its importance and the severity of the errors, it is divided into three classes to be forwarded and processed according to priority.

\section{Critical data}

These are data that are pre-processed results of measurement data that fall into a high-risk category from a predetermined threshold. Immediate transmission of critical data is indispensable for real-time processing of data and the safe passage of other trains on that track.

\section{Important data}

These are data that are pre-processed results of measurement data that fall into the risk category of the predetermined threshold value. Immediate transmission of important data is required for the processing of data and the safe passage of other trains running on that track.

\section{General data}

These are data that are diagnostic data that fall into a non-risk category of the predetermined threshold value. The evaluation of these data is necessary for maintenance planning.

\section{Tool requirements for material test- ing}

For testing at a speed of $200 \mathrm{Km} / \mathrm{h}$, the testing equipment can perform the test, i.e. to produce a continuous, high quality and high-resolution image, it requires a camera that has these criteria. In addition, the camera needs to have a fast recording speed in terms of image/second for which continuous material testing is provided.

For transferring data, a high-speed CAN BUS system is required, which provides fast data transmission without loss of data and redundancy in the case of breakdown or malfunction. 
To evaluate the data in advance, a fast, high-computing onboard computer is required that can be integrated into the rail superstructure. The high-voltage electrical network is electromagnetically shielded and resistant to mechanical impacts. Its storage capacity is high for buffer storage.

\section{Protection of the infrastructure}

To ensure electronic information protection, the security of the transport path and the creation of an endpoint and network security conditions are essential in order for the detected and processed data to be transmitted from the source to the data centre and then to the affected devices. Taking these security considerations into account is indispensable for railways as we are talking about critical infrastructure. Cyber-attacks on critical infrastructures that have occurred on a number of occasions have highlighted the fact that critical infrastructures should be protected against cyber-attacks. This requires the use of technical and procedural rules that ensure principles guaranteeing the protection of information.

\section{Conclusions}

The fracture toughness value is used to define the critical fracture value. If the size of the crack reaches or exceeds the calculated value, it is expected that the rail will be damaged because the Hertzian stress can be extremely high. By continuously monitoring the cracks, we can prevent catastrophic damage.

This monitoring can be effectively supported by automated in-situ visual testing (using a camera). This continuous rail surface monitoring, supported by image analysis software, is necessary for the classification and transmission of the collected surface data according to industry 4.0.

\section{References}

[1] Kazinczy L.: Wheel rail Hertzian contact investigation in case of vehicular train structure. BME, Budapest, 2017. www.epa.uz.ua/00000/00028/00005/pdf/2.pdf

[2] Nyikes Z., Tokody D., Kovács T.: In situ testing of rail damages in accordance with Industry 4.0. Journal of Physics: Conference Series 1045. (2018) 012032, IOP Publishing. http://doi.org/10.1088/17426596/1045/1/012032

[3] Aglan H. A., Fateh M.: Fracture and Fatigue Crack Growth Analysis of Rail Steels, Journal of Mechanics of Materials and Structures 2/2. (2007) 335-346.

https://msp.org/jomms/2007/2-2/jomms-v2n2-p08-p.pdf

[4] Mester Gy. Pletl, S., Pajor G., Rudas I.: Adaptive Control of Robot Manipulators with Fuzzy Supervisor Using Genetic Algorithms. Proceedings of International Conference on Recent Advances in Mechatronics (ICRAM'95) (ed.: Kaynak O.), Istanbul, Turkey, 2. (1995) 661-666.

[5] Mester Gy., Pletl Sz., Pajor G., Jeges Z.: Flexible Planetary Gear Drives in Robotics. Proceedings of the 1992 International Conference on Industrial Electronics, Control, Instrumentation and Automation - Robotics, CIM and Automation, Emerging Technologies (IEEE IECON '92), San Diego, California, USA, 2. (1992) 646-649. http://doi.org/10.1109/IECON.1992.254556

[6] Iantovics B., Crainicu B.: A Distributed Security Approach for Intelligent Mobile Multiagent Systems. In: Advanced Intelligent Computational Technologies and Decision Support Systems. (eds.: Iantovics B., Kountchev R.) Studies in Computational Intelligence, Springer, Cham 486. (2014) 175189.

https://doi.org/10.1007/978-3-319-00467-9_15 